\title{
Relationships among Immune Score, TCF21 Methylation, Risk-factor Level, and Prognosis in Prostate Cancer
}

\author{
Wei Zhang, Zhiyang Huang and Jun Xin \\ Department of Urology, Quanzhou First Hospital Affiliated to Fujian Medical University, Quanzhou, China
}

\begin{abstract}
Objective: To determine whether the immune score and methylation levels of transcription factor 21 (TCF21) could be used as independent diagnostic indicators and prognostic predictors in prostate cancer (PCA).

Study Design: A descriptive study.

Place and Duration of Study: Department of Urology, Quanzhou First Hospital Affiliated to Fujian Medical University, from November 2017 to December 2019.

Methodology: Cases of PCA were for numbers of $\mathrm{CD}^{+}$and $\mathrm{CD} 8^{+} \mathrm{T}$ lymphocytes in the nests and stromal regions of PCA tissues. The percentage of CD8 ${ }^{+} T$ lymphocytes in the peripheral blood was measured. TCF21 methylation was detected. The patient samples were classified according to risk-factor level, the immune score and TCF21 methylation levels were subsequently determined. The time of effective hormone therapy was calculated. The relationships among immune score, TCF21 methylation level, PCA risk-factor level and the time of effective hormone therapy were determined.

Results: There was no significant difference of the proportions of $\mathrm{CD}^{+} \mathrm{T}$ cells in the peripheral blood between different $\mathrm{T}$ stage groups and different Gleason scores groups. The authors found negative linear relationships between immune score and risk-factor level, PSA level and Gleason score $(p<0.001)$. The linear relationship of the immune score with T stage was not significant $(p>0.05)$. The immune score correlated with the time of effective hormone therapy $(p=0.002)$. There was significant correlation between TCF21 methylation and PSA, Gleason score, risk-factor level and immune score $(p<0.05)$. There was also no significant correlation between TCF21 methylation and T stage $(p>0.05)$.

Conclusion: The immune score and TCF21 methylation could be used as independent indicators for the diagnosis and prognosis of PCA.
\end{abstract}

Key Words: Prostatic neoplasms, Immunity cellular, DNA methylation.

How to cite this article: Zhang W, Huang Z, Xin J. Relationships among Immune Score, TCF21 Methylation, Risk-factor Level, and Prognosis in Prostate Cancer. J Coll Physicians Surg Pak 2022; 32(03):319-323.

\section{INTRODUCTION}

Prostate cancer (PCA) is the most frequent cancer affecting men worldwide. ${ }^{1}$ For different risk-factor level of PCA, their optimal treatment methods are different. ${ }^{2,3}$ To judge the risk-factorlevel of PCA is very important.

The factors currently used to predict the risk of PCA are prostate specific antigen (PSA) levels, Gleason score, and clinical TNM stage, which are used to guide treatment and predict prognosis. ${ }^{4}$ However, PSA detection is influenced by several factors, including the time of examination, prostatitis, and urinary retention.

Correspondence to: Jun Xin, Department of Urology, Quanzhou First Hospital Affiliated to Fujian Medical University, No. 248-252 East Street, Licheng District, Quanzhou 362000, People's Republic of China

E-mail: urology@fjmu.edu.cn

Received: August 09, 2021; Revised: November 30, 2021; Accepted: December 24, 2021

DOI: https://doi.org/10.29271/jcpsp.2022.03.319
There is a phenomenon in which patients with similar total Gleason scores have a different prognosis. In clinical staging, the biological characteristics and immunological status of tumor cells are largely ignored. It is an inadequate predictor of recurrence after radical prostatectomy (RP). ${ }^{5}$

In recent years, clinical prognosis prediction models based on immune cell detection and classification have been widely studied, and they are collectively referred to as the immune score. ${ }^{6}$ The immune score is a standard method to quantify the densities of $\mathrm{CD}^{+} \mathrm{T}$ cells (total $\mathrm{T}$ cells) and $\mathrm{CD} 8^{+} \mathrm{T}$ cells (cytotoxic T cells) in the tumor microenvironment, which is important for determining the prognosis of tumors. High expression levels of $\mathrm{CD} 3, \mathrm{CD} 8$ in the centre of the tumor (CT) and invasive margin (IM) were found to be positively correlated with survival and prognosis. ${ }^{7-9}$ The immune score is independent of the TNM stage, and it is used as an independent indicator for predicting overall survival. ${ }^{10,11}$ A study had demonstrated that TCF21 methylation is related to the clonogenic proliferation and migration of renal tumorcel. ${ }^{12}$

TCF21 methylation may be used as a biomarker for determining the diagnosis of renal cancer. ${ }^{13}$ However, the associations 
among the immune score, the methylation status of the TCF21 gene, risk factors, and the prognosis of patients with PCA has not been reported to date.

Therefore, the aim of this study was to build a clinical prognosis prediction model, based on the 'immune score' and 'TCF21 methylation status' in PCA tissues, to provide an experimental basis for establishing effective prognosis prediction tools and clinical treatmentstrategies for PCA.

\section{METHODOLOGY}

Between November 2017 and December 2019, 31 male patients, who were pathologically diagnosed with PCA, at Quanzhou First Hospital Affiliated to Fujian Medical University (Quanzhou, Fujian province, China) were selected for the study. This study was approved by the Ethics Committee of the Quanzhou First Hospital Affiliated to Fujian Medical University. The inclusion criteria were a diagnosis of PCA based on the results of a histological examination; aged 18-70 years; no history of serious heart, lung, liver, kidney, or other diseases; provision of informed consent; good patient compliance; and recipient maxmal androgen blockade (MAB) before castrate-resistant prostate cancer (CRPC). The exclusion criteria were presence of other malignant tumors; serious comorbidities and complications; serious uncontrolled medical diseases or acute infections; restricted legal capabilities; any previous medical history; interference with the test results; or if the study would increase the patient's risk, based on the researcher's judgment.

The observation start time of the research was the time when they received MAB therapy. The observation deadline was December 31, 2020. The end-point event was CRPC. The censored event was the status of loss to follow-up, drug withdrawal, or change of treatment during the follow-up period. For those who were lost to follow-up, the last follow-up time was the end time, for those who stopped the therapy, took the stop MAB time as the end time, for those who changed the treatment method, took the changed time as the end time. The time of effective hormone therapy was the time period from receiving $M A B$ treatment to CRPC.

The patients were also divided into three groups (low, medium, and high risk) according to the risk-factor level for PCA. ${ }^{14}$ Low-risk was the patients' serum PSA levels, which were less than 10 $\mathrm{ng} / \mathrm{mL}$ and T stage wasn't more than T2a and Gleason scores were not more than 6 . High-risk was the patients' serum PSA levels, which were more than $20 \mathrm{ng} / \mathrm{mL}$ or T stage was not less than $\mathrm{T} 2 \mathrm{C}$ or Gleason scores were not less than 8. Others were medium-risk. In the study, 8,10 , and 13 patients were in the lowrisk, medium-risk, and high-risk groups, respectively. Before tissue samples were prepared, written informed consent was obtained from each patient.

For patients who met the inclusion criteria, their relevant clinical information were collected, including age, and ethnicity. Current and past medical history were also collected, including duration of illness, tumor stage and differentiation, treatment, the time of effective hormone therapy, relevant biochemical tests, pathology, and imaging tests. The relevant laboratory tests included routine analysis of blood and urine, liverand kidney function, serum PSA, and peripheral blood $\mathrm{CD}^{+} \mathrm{T}$ lymphocytes and $\mathrm{CD} 8^{+} \mathrm{T}$ lymphocytes. The percentage of $\mathrm{CD} 8^{+} \mathrm{T}$ lymphocytes in the peripheral blood was determined using flow cytometry.

PCA tissue samples were obtained, and the distribution of $\mathrm{CD}^{+}$ and $C D 8^{+} T$ cells in the tissues was evaluated using immunohistochemical staining. Briefly, the tissue sections were subjected to microwave antigen retrieval, incubated with the primary antibodies overnightat $4^{\circ} \mathrm{C}$, and then with a horseradish peroxidase-conjugated secondary antibody. Color development was performed using DAB, and then, the sections were counterstained using hematoxylin and sealed with neutral gum. For the negative controls, homotypic control antibodies were substituted for the primary antibodies. Cells with brown cell membranes and T cell-like morphology were determined to be $\mathrm{CD}^{+}{ }^{+}$or $\mathrm{CD} 8^{+}$Tcells.

Immunohistochemistry was used to detect the distribution of $\mathrm{CD}^{+}$and $\mathrm{CD}^{+} \mathrm{T}$ cells in the prostate tissues. Whole sections were first observed under low-power magnification $(\times 100)$ to identify the cancerous nests and mesenchymal regions in the PCA tissues. Then, five independent, high-power magnification $(\times 400)$ fields of view were selected in the cancerous nests and mesenchymal regions with an area of $0.04 \mathrm{~mm}^{2}$. The number of cells/field of view was evaluated separately by two independent researchers. To reduce subjective differences in experimenter counts, the mean of the two individuals' count data was used in the statistical analysis.

The numbers of $\mathrm{CD}^{+}$and $\mathrm{CD} 8^{+} \mathrm{T}$ cells in the tumor areas were counted, and the ratio of $\mathrm{CD}^{+} / \mathrm{CD}^{+} T$ cells was calculated based on the least $P$-value method, using the X-tile software (version 3.6.1). For $C D 8^{+}$and $C D 3^{+} T$ cells, different values were used as cutoffs for the cardinal test, and when the $P$-value reached the minimum value, this was determined to be the optimal critical value, i.e., at the minimum P-value method (also known as the maximum cardinal statistic method). The patients were then divided into two groups: the high-density and low-density groups. High-density was scored as 1 point; whereas, low-density was scored as 0 points. Finally, the $\mathrm{CD}^{+}$and $\mathrm{CD} 8^{+}$cells in the cancer nest area (CT) and the density scores for the IM (interstitial zone) region were summed to obtain a total immune score $(0-4){ }^{7}$

Paraffin-embedded tissue sections from 31 PCA patients were selected randomly, and nMSP was used to determine the methylation level in the samples. Samples of bisulfite-modified DNA were used as the template, and the methylation level in each sample was detected using the SYBR Green fluorescent dye. Experiments were performed in triplicate. The results are expressed as mean $\pm \mathrm{SD}$.

In addition, to search for novel markers of PCA, the authors focused on gene methylation. First, based on a search of the TCGA online database (http://www.cbioportal.org/), methylation chip data and a literature review, we chose to evaluate the methylation of a significant and relatively novel gene, TCF21 (Figure 1). 

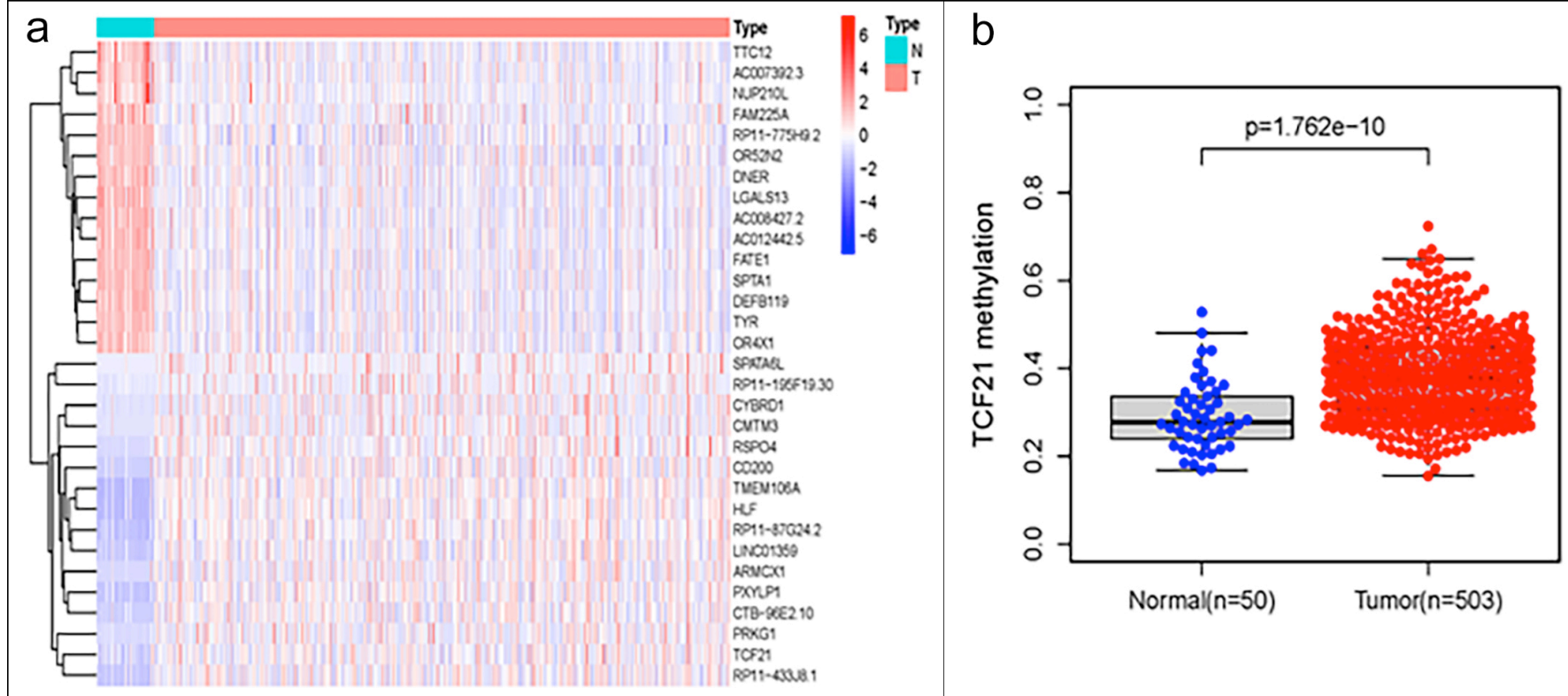

Figure 1: (a) The top 31 different genes' methylation between para-cancerous and PCA tissues from TCGA database. (b) The bar chart shows TCF21 gene methylation between para-cancerous and PCA tissues.

Table I: Correlation analysis of TCF21 methylation levels and clinical parameters in the PCA.

\begin{tabular}{|l|l|c|c|c|c|c|}
\hline & & T-stage & PSA & Gleason score & Risk factor level & Immune score \\
\hline \multirow{2}{*}{ Spearman Rho } & Correlation coefficient & 0.241 & 0.410 & 0.453 & 0.459 & -0.395 \\
\cline { 2 - 7 } & p-value & 0.192 & 0.022 & 0.010 & 0.009 & 0.028 \\
\hline
\end{tabular}

The peripheral blood $\mathrm{CD}^{+} \mathrm{T}$ cell ratio was expressed as mean and standard deviation, and the mean values among different $T$ stage groups, different Gleason scores groups were respectively evaluated, using a randomised block design ANOVA. If ANOVA test was significant $(p<0.05)$, the mean values of the group were compared using the SNK post-hoc test.

Spearman's correlation was used to analyse the correlations between immune score and PSA level, T stage, Gleason score and risk-factor level in PCA patients. Spearman's correlation was further used to analyse the correlation between the methylation level of the TCF21 gene in PCA tissues and other clinical parameters (T stage, PSA, Gleason score, risk-factor level and immune score). Log-Rank test in KaplanMeier was used to compare the difference in CRPC time among different immune score groups.

All statistical analyses were performed using SPSS version 19.0. A $P$-value less than 0.05 was considered significant, and a $P$-value less than 0.01 was considered highly significant.

\section{RESULTS}

Flow cytometry was used to detect T cells in the peripheral blood of patients with PCA. The peripheral blood $\mathrm{CD} 8^{+} \mathrm{T}$ cell ratio ( $\mathrm{CD} 8^{+} \mathrm{T}$ cells to $\mathrm{CD}^{+} \mathrm{T}$ cells) was $60.13 \% \pm 22.33 \%$. The mean values among different $T$ stage groups, different Gleason scores groups were respectively evaluated using a randomised block design ANOVA. The results showed that $T$ stage and Gleason score had no effect on the percentage of CD8 ${ }^{+} T$ cells in peripheral blood $(p=0.579$, and 0.458 , respectively).

The association of the calculated immune score with PCA risk-factor level, PSA level, Gleason score, and T stage was assessed using Bivariate Correlation analysis. The results showed that the immune score was correlated with PCA risk-factor level $(p<0.001, r=-0.663)$, PSA level $(p<0.001, r=$ -0.664), and Gleason score ( $p<0.001, r=-0.614)$; however, it was not significantly correlated with $T$ grade $(p=0.078$, $r=-0.322$ ).

The Kaplan-Meier analysis of the time of effective hormone therapy showed that the impact of immune score on the time of effective hormone therapy is statistically significant $(p=0.002, \chi 2=16.650)$. The correlation coefficients for TCF21 gene methylation level with PSA, Gleason score, risk-factor level and immune score were 0.410, 0.453, 0.459 and -0.395 , respectively. These correlations had statistical significance ( $p=0.022,0.010,0.009$, and 0.028 , respectively). The correlation coefficients for TCF21 gene methylation level with $T$ stage was 0.241 , the correlation had no statistical significance ( $p>0.05$, Table I).

\section{DISCUSSION}

PCA is the most frequent cancer affecting men worldwide ${ }^{1}$, The variability in patient outcomes based on current risk prediction methods, it is important to develop a new prog- 
nostic evaluation system for early diagnosis of PCA to provide appropriate clinical treatment. In recent years, more and more literature reports that immunity is closely related to the occurrence and development of prostate cancer. Zehua et al. illustrated the immune infiltration phenotypes strongly related to the poor prognosis of prostate adenocarcinoma patients, and highlighted the potential of the immune prognostic model to identify unfavorable tumor features. ${ }^{15}$ Prostate cancer patients with mutations in some immune-related genes together have a worse prognosis. ${ }^{16} \mathrm{CD}^{+} \mathrm{T}$ lymphocytes are important immune cells. CD8 is the main surface marker of cytotoxic T cells (CTL), which can kill tumor cells. Sotosek et al. found that there was no significant difference in the number of $\mathrm{CD}^{+} \mathrm{T}$ lymphocytes in the peripheral blood of patients with benign prostatic hyperplasia and those with PCA. ${ }^{17}$ Similarly, in the present study, the results showed that there was no significant difference in the number of $C D 8^{+} \mathrm{T}$ cells in the peripheral blood of patients with PCA.

In terms of the relationship between the immune score and prognosis, Petitprez et al. found that the number of CD8+ T lymphocytes in the tumor tissues of PCA patients with lymph node metastasis was associated with a risk of clinical progression. ${ }^{18}$ Other related studies showed that the number of CD8+ T lymphocytes in PCA tissue is related to the biochemical recurrence of PCA. ${ }^{19}$ In addition, Nessn et al. and others found that the number of CD8+ T lymphocytes in the epithelial region of PCA was negatively correlated with the biochemical recurrence of PCA. ${ }^{20}$ The number of CD4+ T lymphocytes in PCA tissue was positively correlated with the risk of death in patients with PCA and was an independent predictor of prognosis. ${ }^{21}$ The immune score is important in evaluating prognosis, as the standard method to quantify the density of $\mathrm{CD}^{+} \mathrm{T}$ cells and $\mathrm{CD}^{+} \mathrm{T}$ cells in the tumor microenvironment. As effector cells, $C D 8^{+} \mathrm{T}$ cells have good prognostic value in esophageal, gastric, and colorectal cancer, as well as other tumors. ${ }^{22}$ The PCA risk score is a comprehensive indicator that can guide treatment and predict prognosis. ${ }^{23}$ Patients with a high immune score displayed better overall survival (OS) than patients with a low immune score. ${ }^{24}$ It demonstrated that immune score is a protective factor, which is similar to this study. The preliminary study showed a correlation between immune score and risk-factor level, PSA level, Gleason score and time of effective hormone therapy. This shows that the immune score can be used to predict the prognosis of patients with PCA, thereby guiding the treatment.

Because most of the patients in this study are still alive and have not reached the five-year follow-up time point and most are treated conservatively, the os and disease-free survival cannot be calculated. But the time of effective hormone therapy, which means the time period from receiving $M A B$ treatment to $C R P C$, is calculated to indirectly reflect the prognosis of prostate cancer. However, the corre- lation between the immune score in the PCA and overall survival requires further study.

The tumor suppressor gene TCF21 encodes the transcription factor 21, which is involved in the occurrence and development of tumors. ${ }^{12,13}$ Methyltransferases catalyse the addition of a methyl group to the cytosine of CpG dinucleotides in DNA to form 5-methylcytosine. DNA methylation can cause changes in DNA conformation, DNA stability, and DNA-protein interactions, resulting in gene inactivation and silencing, or downregulation of gene expression, thus affecting gene function. TCF21 methylation is the main cause of TCF21 expression downregulation, which is closely related to tumor development. In this study, nMSP was used to detect TCF21 methylation levels in patients with PCA. The results showed that TCF21 methylation was significantly correlated with PSA, Gleason score, risk-factorlevel and immune score in patients with PCA. This shows that TCF21 methylation can also be used to predict the prognosis and guide the treatment of patients with PCA.

\section{CONCLUSION}

This study is a preliminary study of a predictive model for the clinical prognosis of PCA using an immune score and TCF21 methylation. It lays the foundation for the early diagnosis, treatment, and survival prognosis of PCA through the detection of immune score and DNA methylation in PCA tissues to generate a new prognosis-prediction model.

\section{FUNDING:}

This study was supported by the Startup Fund for Scientific Research of Fujian Medical University (No. 2017XQ1172) and Science and Technology Projects for Quanzhou (No. 2018N075S).

\section{ETHICAL APPROVAL:}

The study has been approved by the Ethics Committee of Quanzhou First Hospital Affiliated to Fujian Medical University.

\section{PATIENTS' CONSENT:}

Written consents were obtained from all patients.

\section{AVAILABILITY OF DATA AND MATERIALS:}

The datasets generated during and/or analysed during the current study are available from the corresponding author on reasonable request.

\section{CONFLICT OF INTEREST:}

The authors declared no conflict of interest.

\section{AUTHORS' CONTRIBUTION:}

WZ: Designed the study, completed the experiment and wrote the article.

$\mathrm{ZH}$ : Revised the article and optimised the language.

JX: Directed the conception of the work and did the analysis.

All authors read and approved the final manuscript. 


\section{REFERENCES}

1. Siegel RL, Miller KD, Jemal A. Cancer Statistics, 2017. CA Cancer J Clin 2017; 67(1):7-30. doi: 10.3322/caac.21387.

2. Ansmann L, Winter N, Ernstmann N, Heidenreich A, Weissbach L, Herden J. Health-related quality of life in active surveillance and radical prostatectomy for low-risk prostate cancer: A prospective observational study (HAROW Hormonal therapy, active surveillance, radiation, operation, watchful waiting). BJU Int 2018; 122(3):401-10. doi: 10.1111/bju.14215.

3. Salmasi A, Faiena I, Wu J, Sisk AE Jr., Sachveda A, Vandel JJ, et al. Radical prostatectomy then and now: Surgical overtreatment of prostate cancer is declining from 2009 to 2016 at a tertiary referral center. Urol Oncol 2018; 36(9):401.e19-.e25. doi: 10.1016/j.urolonc.2018.06.006.

4. Grignon DJ. Prostate cancer reporting and staging: Needle biopsy and radical prostatectomy specimens. Mod Pathol 2018; 31(S1):S96-109. doi: 10.1038/modpathol.2017.167.

5. Saoud R, Heidar NA, Cimadamore A, Paner GP. Incorporating prognostic biomarkers into risk assessment models and tnm staging for prostate cancer. Cells 2020; 9(9):2116. doi: 10.3390/cells9092116.

6. Yuan W, Cai W, Huang X, Peng S. Prognostic value of immune scores in the microenvironment of colorectal cancer. Oncol Lett 2020; 20(5):256. doi: 10.3892/ol.2020. 12119.

7. Anitei MG, Zeitoun G, Mlecnik B, Marliot F, Haicheur N, Todosi AM, et al. Prognostic and predictive values of the immunoscore in patients with rectal cancer. Clin Cancer Res 2014; 20(7):1891-9. doi: 10.1158/1078-0432.CCR-13-2830.

8. Malka D, Lièvre A, André T, Taïeb J, Ducreux M, Bibeau F. Immune scores in colorectal cancer: Where are we? Eur J Cancer 2020; 140:105-18. doi: 10.1016/j.ejca.2020.08.024.

9. Idos GE, Kwok J, Bonthala N, Kysh L, Gruber SB, Qu C. The prognostic implications of tumor infiltrating lymphocytes in colorectal cancer: A systematic review and meta-analysis. Sci Rep 2020; 10(1):3360. doi: 10.1038/s41598-02060255-4.

10. Galon J, Mlecnik B, Bindea G, Angell HK, Berger A, Lagorce $C$, et al. Towards the introduction of the 'Immunoscore' in the classification of malignant tumours. J Pathol 2014; 232(2): 199-209. doi: 10.1002/path.4287.

11. Galon J, Pagès $F$, Marincola FM, Thurin M, Trinchieri G, Fox $\mathrm{BA}$, et al. The immune score as a new possible approach for the classification of cancer. J Transl Med 2012; 10:1. doi: 10.1186/1479-5876-10-1.

12. Gooskens SL, Klasson TD, Gremmels H, Logister I, Pieters R, Perlman EJ, et al. TCF21 hypermethylation regulates renal tumor cell clonogenic proliferation and migration. Mol Oncol 2018; 12(2):166-79. doi: 10.1002/1878-0261.12149.

13. Costa VL, Henrique $R$, Danielsen SA, Eknaes M, Patrício $P$, Morais $A$, et al. TCF21 and PCDH17 methylation: An innovative panel of biomarkers for a simultaneous detection of urological cancers. Epigenetics 2011; 6(9):1120-30. doi: 10.4161/epi.6.9.16376.

14. D'Amico AV, Whittington R, Malkowicz SB, Schultz D, Blank $\mathrm{K}$, Broderick GA, et al. Biochemical outcome after radical prostatectomy, external beam radiation therapy, or interstitial radiation therapy for clinically localised prostate cancer. Jama 1998; 280(11):969-74. doi: 10.1001/jama.280.11.969.

15. Ma Z, Cheng X, Yue T, Shangguan X, Xin Z, Zhang W, et al. Immune infiltration phenotypes of prostate adenocarcinoma and their clinical implications. Cancer Med 2021; 10(15): 5358-74. doi: 10.1002/cam4.4063.

16. Lv D, Wu X, Chen X, Yang S, Chen W, Wang M, et al. A novel immune-related gene-based prognostic signature to predict biochemical recurrence in patients with prostate cancer after radical prostatectomy. Cancer Immunol Immunother 2021; 70(12):3587-602. doi: 10.1007/s00262-021-02923-6.

17. Sotosek S, Sotosek Tokmadzic V, Mrakovcic-Sutic I, Tomas MI, Dominovic M, Tulic V, et al. Comparative study of frequency of different lymphocytes subpopulation in peripheral blood of patients with prostate cancer and benign prostatic hyperplasia. Wien Klin Wochenschr 2011; 123(23-24): 718-25. doi: 10.1007/s00508-011-0096-7

18. Petitprez F, Fossati N, Vano Y, Freschi M, Becht E, Lucianò R, et al. PD-L1 Expression and CD8 (+) T-cell infiltrate are associated with clinical progression in patients with node-positive prostate cancer. Eur Urol Focus 2019; 5(2):192-6. doi: 10.1016/j.euf.2017.05.013.

19. Zeigler-Johnson C, Morales KH, Lal P, Feldman M. The relationship between obesity, prostate tumor infiltrating lymphocytes and macrophages, and biochemical failure. PLoS One 2016; 11(8):e0159109. doi: 10.1371/journal.pone.015 9109.

20. Ness N, Andersen S, Valkov A, Nordby Y, Donnem T, Al-Saad $S$, et al. Infiltration of CD8+ lymphocytes is an independent prognostic factor of biochemical failure-free survival in prostate cancer. Prostate 2014; 74(14):1452-61. doi: 10.1002/pros.22862.

21. Davidsson S, Ohlson AL, Andersson SO, Fall K, Meisner A, Fiorentino $M$, et al. CD4 helper T cells, CD8 cytotoxic T cells, and $\mathrm{FOXP3}(+)$ regulatory $T$ cells with respect to lethal prostate cancer. Mod Pathol 2013; 26(3):448-55. doi: 10.1038/modpathol.2012.164.

22. Fridman WH, Pagès $F$, Sautès-Fridman $C$, Galon J. The immune contexture in human tumours: impact on clinical outcome. Nat Rev Cancer 2012; 12(4):298-306. doi: $10.1038 / \mathrm{nrc} 3245$.

23. Huang J WJKC. Guidelines for diagnosis and treatment of urology and andrology diseases in China. Science Press 2019.

24. Hao D, Liu J, Chen M, Li J, Wang L, Li X, et al. Immunogenomic analyses of advanced serous ovarian cancer reveal immune score is a strong prognostic factor and an indicator of chemosensitivity. Clin Cancer Res 2018; 24(15):3560-71. doi: 10.1158/1078-0432.CCR-17-3862. 